S. Ikeda

Nagoya Math. J.

Vol. 102 (1986), 135-154

\title{
ON THE GORENSTEINNESS OF REES ALGEBRAS OVER LOCAL RINGS
}

\author{
SHIN IKEDA
}

\section{Introduction}

Let $(A, m, k)$ be a Noetherian local ring and $I$ an ideal of $A$. We set $R(I)=\oplus_{n \geq 0} I^{n}$ and call this graded $A$-algebra the Rees algebra of $I$. The importance of the Rees algebra $R(I)$ is in the fact that Proj $R(I)$ is the blowing up of $\operatorname{Spec} A$ with center in $V(I)$. The Cohen-Macaulayness of Rees algebras was studied by many mathematicians. In [GS] S. Goto any $\mathrm{Y}$. Shimoda gave a criterion for $R(m)$ to be Cohen-Macaulay under the assumption that $A$ is Cohen-Macaulay. Their results have been generalized to $R(I)$ in $[\mathrm{HI}]$.

Let grade $(I) \geq 2$. The purpose of this paper is to characterize the Gorensteinness of $R(I)$ in terms of canonical modules of $A$ and the associated graded ring $G(I)=\oplus_{n \geq 0} I^{n} / I^{n+1}$. The notion of canonical modules of local rings plays an important role in the homological theory of local rings, cf. [HK]. The canonical modules of graded rings defined over a field were introduced and studied extensively in [GW]. In Section 1 we introduce the notion of canonical modules of graded rings defined over a local ring. Our definition of canonical modules coincides with that of [GW] if the local ring is a field. In Section 2 we collect several facts about the behaviour of the local cohomology modules of Rees algebras. Section 3 will be devoted to the proof of our criterion of the Gorensteinness of $R(I)$ and to the construction of an example of a local ring $(A, m, k)$ such that $R(m)$ is Gorenstein but $A$ is not Cohen-Macaulay.

\section{§1. Local cohomology of graded rings}

In this section we give a brief summary of the theory of local cohomology and duality of graded rings.

Let $R=\oplus_{n \in Z} R_{n}$ be a Noetherian graded ring and let $M, N$ be graded

Received January 29, 1985. 
$R$-modules. Let us denote the category of graded $R$-modules by $M_{H}(R)$. A morphism in $M_{H}(R) f: M \rightarrow N$ is an $R$-linear map such that $f\left(M_{n}\right) \subset N_{n}$ for all $n \in Z$. Let $n \in Z$. We denote by $M(n)$ the graded $R$-module whose grading is defined by $M(n)_{m}=M_{n+m}$ for all $m \in Z$. Let $\mathscr{H}_{o m_{R}}(M, N)_{n}$ be the abelian group of all homomorphisms from $M$ into $N(n)$ in $M_{H}(R)$. Let $\mathscr{H}_{o m_{R}}(M, N)=\oplus_{n \in Z} \mathscr{H}_{o m_{R}}(M, N)_{n}$. Then $\mathscr{H}_{o m_{R}}(M, N)$ is a graded $R$ module whose homogeneous component of degree $n$ is $\mathscr{H}_{o m_{R}}(M, N)_{n}$. A graded $R$-module $E$ is injective (resp. projective) in $M_{H}(R)$ if the functor $\mathscr{H}_{o m_{R}}(, E)$ (resp. $\left.\mathscr{H}_{o m_{R}}(E),\right)$ from $M_{H}(R)$ into itself is an exact functor.

The tensor product $M \otimes_{R} N$ is a graded $R$-module whose $n$-th homogeneous component is the abelian group generated by the elements of the form $x \otimes y$ with $x \in M_{i}, y \in N_{j}$ and $i+j=n$.

The category $M_{H}(R)$ is an abelian category with enough injectives (cf. [Gr $],(1,10))$. A homomorphism $f: M \rightarrow N$ in $M_{H}(R)$ is called essential if $f$ is an injection and for any non-trivial graded $R$-submodule $L$ of $N$ we have $f(M) \cap L \neq 0$. The injective envelope of a graded $R$-module $M$ is an injective object $\mathscr{E}_{R}(M)$ of $M_{H}(R)$ with an essential homomorphism $M \rightarrow \mathscr{E}_{R}(M)$ in $M_{H}(R)$.

The following proposition describes the structure of injective objects in $M_{H}(R)$.

Proposition (1.1). (1) Let $M$ be a graded $R$-module. Then

$$
\operatorname{Ass}_{R}\left(\mathscr{E}_{R}(M)\right)=\operatorname{Ass}_{R}(M) .
$$

(2) Let $E$ be an injective object of $M_{H}(R)$. Then $E$ is indecomposable if and only if $E=\mathscr{E}_{R}(R / p)(n)$ for some homogeneous prime ideal of $R$ and for some $n \in Z$.

(3) Every injective object of $M_{H}(R)$ can be decomposed into a direct sum of indecomposable injective objects of $M_{H}(R)$. This decomposition is unique up to isomorphism.

Proof. This is [GW], (1.2.1).

For $i \geq 0$ the functor $\mathscr{E} x t_{R}^{i}($,$) is defined to be the i$-th derived functor of the functor $\mathscr{H}_{o m_{R}}($,$) . Suppose that M$ is a finitely generated graded $R$-module. Then $\mathscr{H}_{o m_{R}}(M, N)=\operatorname{Hom}_{R}(M, N)$ as underlying $R$-modules. Hence $\mathscr{E}_{x t_{R}}^{i}(M, N)=\operatorname{Ext}_{R}^{i}(M, N)$ for all $i \geq 0$. For any $p \in \operatorname{Spec}(R)$ and for any $R$-module $L$ we define

$$
\mu^{i}(p, L)=\operatorname{dim}_{k(p)} \operatorname{Ext}_{R_{p}}^{i}\left(k(p), L_{p}\right),
$$


where $k(p)=R_{p} / p R_{p}$, and call this number the $i$-th Bass number of $M$ at $p$ (cf. [B]).

Proposition (1.2). Let $M$ be a graded $R$-module and let

$$
0 \rightarrow M \rightarrow I^{0} \rightarrow I^{1} \rightarrow \cdots \rightarrow I^{n} \rightarrow I^{n+1} \rightarrow \cdots
$$

be a minimal injective resolution of $M$ in $M_{H}(R)$. Then for any homogeneous prime ideal $p$ and for any integer $i \geq 0, \mu^{i}(p, M)$ is equal to the number of the graded $R$-modules of the form $\mathscr{E}_{R}(R / p)(n)$ which appear in $I^{i}$ as direct summands.

Proof. This is [GW], (1.2.4).

In this paper a Noetherian graded $R$ is called defined over a local ring if $R_{0}$ is a Noetherian local ring and $R_{n}=0$ for $n<0$. If $R$ is defined over a local ring we denote the graded ring $R \otimes_{R_{0}} \hat{R}_{0}$ by $\hat{R}$, where $\hat{R}_{0}$ is the completion of $R_{0}$. In the rest of this section $R$ denotes a graded ring defined over a local ring $\left(R_{0}, m_{0}, k\right)$ and $M$ denotes the maximal homogeneous ideal of $R$. $R$ can be regarded as a graded $R_{0}$-module in a natural way. Let $E_{R_{0}}$ be the injective envelope of $k$ as an $R_{0}$-module. We denote by $\mathscr{E}_{R_{0}}$ the graded $R$-module whose underlying $R_{0}$-module is $E_{R_{0}}$ and whose grading is given by $\left[\mathscr{E}_{R_{0}}\right]_{0}=E_{R_{0}}$ and $\left[\mathscr{E}_{R_{0}}\right]_{n}=0$ for $n \neq 0$.

Definition (1.3). $\quad \mathscr{E}_{R}(k)=\mathscr{H}_{\mathrm{om}_{R_{0}}}\left(R, \mathscr{E}_{R_{0}}\right)$.

Proposition (1.4). (1) $\mathscr{E}_{R}(k)$ is the injective envelope of $R / M$ in $M_{H}(R)$.

(2) $\mathscr{H}_{\mathrm{om}_{R}}\left(\mathscr{E}_{R}(k), \mathscr{E}_{R}(k)\right)=R \otimes_{R_{0}} \hat{R}_{0}$, where $\hat{R}_{0}$ is the completion of $R_{0}$.

Proof. (1) As in the non-graded case, in order to show that $\mathscr{E}_{R}(k)$ is injective in $M_{H}(R)$ it is enough to show that for any homogeneous ideal of $R$ and for any integer $n$ every homomorphism $f: I(n) \rightarrow \mathscr{E}_{R}(k)$ can be extended to a homomorphism $f^{\prime}: R(n) \rightarrow \mathscr{E}_{R}(k)$. Since

$$
\mathscr{H}_{o m_{R_{0}}}\left(R, \mathscr{E}_{R_{0}}\right) \subset \operatorname{Hom}_{R_{0}}\left(R, E_{R_{0}}\right)=\prod_{i \in Z} \operatorname{Hom}_{R_{0}}\left(R_{i}, E_{R_{0}}\right),
$$

and since $\operatorname{Hom}_{R_{0}}\left(R, E_{R_{0}}\right)$ is an injective $R$-module $f$ can be extended to an $R$-homomorphism $f^{\prime \prime}: R \rightarrow \operatorname{Hom}_{R_{0}}\left(R, E_{R_{0}}\right)$.

Let $f^{\prime \prime}(1)=\left(g_{i}\right)_{i \in Z}$, where $g_{i} \in \operatorname{Hom}_{R_{0}}\left(R_{-i}, E_{R_{0}}\right)$. Since $f$ is homogeneous for any homogeneous element $x \in I$ we have $x g_{j}=0$ for $j \neq-n$. This shows that the homomorphism $f^{\prime}$ in $M_{H}(R)$ defined by $f^{\prime}(1)=g_{-n} \in$ 
$\operatorname{Hom}_{R_{0}}\left(R_{n}, E_{R_{0}}\right)$ extends $f$. It is not difficult to show that $\operatorname{Supp}\left(\mathscr{E}_{R}(k)\right)=M$. Moreover we have

$$
\begin{aligned}
\mathscr{H}_{o m_{R}}\left(R / M, \mathscr{E}_{R}(k)\right) & =\mathscr{H}_{o m_{R}}\left(R / M, \mathscr{H}_{o m_{R_{0}}}\left(R, \mathscr{E}_{R_{0}}\right)\right) \\
& =\mathscr{H}_{o m_{R_{0}}}\left(R / M, \mathscr{E}_{R_{0}}\right) \\
& =k .
\end{aligned}
$$

This shows that $\mathscr{E}_{R}(k)$ is the injective envelope of $R / M$ in $M_{H}(R)$.

$$
\begin{aligned}
& \mathscr{H}_{o m_{R}}\left(\mathscr{E}_{R}(k), \mathscr{E}_{R}(k)\right)=\mathscr{H}_{o m_{R}}\left(\mathscr{E}_{R}(k), \mathscr{H}_{o m_{R_{0}}}\left(R, \mathscr{E}_{R_{0}}\right)\right) \\
& =\mathscr{H}_{o m_{R_{0}}}\left(\mathscr{E}_{R}(k), \mathscr{E}_{R_{0}}\right) \\
& =\mathscr{H}_{o m_{R_{0}}}\left(\mathscr{H}_{o m_{R_{0}}}\left(R, \mathscr{E}_{R_{0}}\right), \mathscr{E}_{R_{0}}\right) \\
& =\bigoplus_{n \in Z} \operatorname{Hom}_{R_{0}}\left(\operatorname{Hom}_{R_{0}}\left(R_{n}, E_{R_{0}}\right), E_{R_{0}}\right) \\
& =\bigoplus_{n \in Z} R_{n} \otimes_{R_{0}} \hat{R}_{0} \\
& =R \otimes_{R_{0}} \hat{R}_{0} \text {. }
\end{aligned}
$$

Proposition (1.5). Let $R$ be a graded ring defined over a complete local ring $R_{0}$ and $N$ a graded $R$-module. Then, we have:

(1) If $N$ is Noetherian (resp. Artinian) $\mathscr{H}_{o m_{R}}\left(N, \mathscr{E}_{R}(k)\right.$ ) is Artinian (resp. Noetherian).

(2) If $N$ is Noetherian or Artinian

$$
\mathscr{H}_{o m_{R}}\left(\mathscr{H}_{\mathrm{om}_{R}}\left(N, \mathscr{E}_{R}(k)\right), \mathscr{E}_{R}(k)\right)=N .
$$

Proof. Using Proposition (1.4) this can be proved as in [M].

For every integer $i \geq 0$ we put

$$
\mathscr{H}_{M}^{i}(\quad)=\underline{\lim _{n}} \mathscr{E}_{x t_{R}^{i}}\left(R / M^{n}, \quad\right)
$$

and call it the $i$-th local cohomology functor, where $R$ is a graded ring defined over a local ring and $M$ is the maximal homogeneous ideal of $R$. $\mathscr{H}_{M}^{i}()$ is the $i$-th derived functor of $\mathscr{H}_{M}^{0}\left(\right.$ ) (cf. $\left[\mathrm{Gr}_{2}\right]$ and [HK]).

Definition (1.6). Suppose that $R_{0}$ is complete. We put

$$
\mathscr{K}_{R}=\mathscr{H}_{o m_{R}}\left(\mathscr{H}_{M}^{d}(R), \mathscr{E}_{R}(k)\right),
$$

where $d=\operatorname{dim} R$, and call this graded $R$-module the canonical module of $R$.

If $R_{0}$ is not complete a graded $R$-module $\mathscr{K}_{R}$ is a canonical module of $R$ if there is an isomorphism in $M_{H}(\hat{R}) \mathscr{K}_{\hat{R}}=\mathscr{K}_{R} \otimes_{R} \hat{R}$. 
Proposition (1.7). If there is a canonical module of $R$ it is a finitely generated $R$-module and unique up to isomorphisms.

Proof. Since $\hat{R}$ is faithfully flat over $R$ it is sufficient to show that $\mathscr{K}_{\hat{R}}$ is finitely generated. But this follows from Proposition (1.5). For the proof of the uniqueness it is enough to show that if $K$ and $L$ are finitely generated graded $R$-modules such that $K \otimes_{R} \hat{R}=L \otimes_{R} \hat{R}$ then $K=L$. Let $f \in \mathscr{H}_{\mathrm{oo}} \hat{\hat{R}}\left(K \otimes_{R} \hat{R}, L \otimes_{R} \hat{R}\right)_{0}$ be an isomorphism. Since $\hat{R}$ is flat over $R$ and $K$ is finitely generated over $R$ one gets

$$
\begin{aligned}
\mathscr{H}_{o m_{\hat{R}}}\left(K \otimes_{R} \hat{R}, L \otimes_{R} \hat{R}\right) & =\mathscr{H}_{o m_{R}}(K, L) \otimes_{R} \hat{R} \\
& =\mathscr{H}_{o m_{R}}(K, L) \otimes_{R_{0}} \hat{R}_{0}
\end{aligned}
$$

which implies that $\mathscr{H}_{\mathrm{om}}\left(K \otimes_{R} \hat{R}, L \otimes_{R} \hat{R}\right)_{0}$ is the completion of $\mathscr{H}_{\mathrm{H}} \mathrm{om}_{R}(K, L)_{0}$ since $\mathscr{H}_{o m_{R}}(K, L)_{0}$ is a finitely generated $R_{0}$-module. Let $\mathscr{H}_{o m_{R}}(K, L)_{0}^{\wedge}$ be the $m_{0}$-adic completion of $\mathscr{H}_{o m_{R}}(K, L)_{0}$. For any integer $n>0$ there is a homomorphism $f_{n} \in \mathscr{H}_{o m_{R}}(K, L)_{0}$ such that $f-f_{n} \in m_{0}^{n} \mathscr{H}_{o m_{R}}(K, L)_{0}^{\hat{0}}$. By assumption $f_{n}$ induces an isomorphism $\bar{f}_{n}: K / m_{0}^{n} K \rightarrow L / m_{0}^{n} L$. Hence $f_{n}$ is a surjective homomorphism. Since $K / M K$ and $L / M L$ are isomorphic there exist finitely generated graded free $R$-modules $F$ and $G$ of the same rank $\operatorname{dim}_{k} K / M K$ such that there are surjective homomorphisms in $M_{H}(R)$ $g: F \rightarrow K$ and $h: G \rightarrow L$. Let $S=\operatorname{Ker}(g)$ and $T=\operatorname{Ker}(h)$. We get a commutative diagram with exact rows

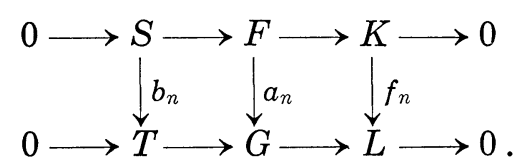

$a_{n}$ is an isomorphism since $F$ and $G$ are free $R$-modules of the same rank. Since $\bar{f}_{n}$ is an isomorphism from (I) we get

$$
T \subset b_{n}(S)+m_{0}^{n} G \cap T .
$$

By Artin-Rees lemma there is an integer $r>0$ such that

$$
m_{0}^{n} G \cap T=m_{0}^{n-r}\left(m_{0}^{r} G \cap T\right) \quad \text { for } n>r .
$$

Therefore we get $T \subset b_{n}(S)+m_{0} T$ for $n>r$. By Nakayama's lemma $T=b_{n}(S)$. From (I) one knows that $f_{n}$ is an isomorphism.

Let us recall that $R$ is Cohen-Macaulay (resp. Gorenstein) if and only if $R_{M}$ is Cohen-Macaulay (resp. Gorenstein), see [AG], [MR] and [GW]. 
Proposition (1.8). Let $d=\operatorname{dim} R$ and assume that $R_{0}$ is complete. Then $R$ is Cohen-Macaulay if and only if for any finitely generate graded $R$-module $N$ and for all $i \geq 0$ we have

$$
\mathscr{H}_{o m_{R}}\left(\mathscr{H}_{M}^{i}(N), \mathscr{E}_{R}(k)\right)=\mathscr{E}_{x t_{R}^{d-i}}\left(N, \mathscr{K}_{R}\right) .
$$

Proof. Suppose that $R$ is Cohen-Macaulay. We will show that the functor $T^{i}()=\mathscr{H}_{o m_{R}}\left(\mathscr{H}_{M}^{d-i}(), \mathscr{E}_{R}(k)\right)$ is the $i$-th derived functor of $\mathscr{H}_{o m_{R}}\left(, \mathscr{K}_{R}\right)$. We must show that

(1) from the short exact sequence $0 \rightarrow N^{\prime} \rightarrow N \rightarrow N^{\prime \prime} \rightarrow 0$ we obtain the long exact sequence

$$
0 \rightarrow T^{0}\left(N^{\prime \prime}\right) \rightarrow T^{0}(N) \rightarrow T^{0}\left(N^{\prime}\right) \rightarrow T^{1}\left(N^{\prime \prime}\right) \rightarrow T^{1}(N) \rightarrow T^{1}\left(N^{\prime}\right) \rightarrow \cdots
$$

(2) $T^{i}(R)=0$ for $i>0$.

Since $\mathscr{E}_{R}(k)$ is an injective object in $M_{H}(R)$ (1) follows from the long exact sequence of the local cohomology. (2) follows from the fact that for any graded $R$-module $N \mathscr{H}_{o m_{R}}\left(N, \mathscr{E}_{R}(k)\right)=0$ if and only if $N=0$. The converse is immediate.

Proposition (1.9). Suppose that $R$ is Cohen-Macaulay. Then $R$ is Gorenstein if and only if $R$ has a canonical module $\mathscr{K}_{R}$ and $\mathscr{K}_{R}=R(n)$ for some $n \in Z$.

Proof. Recall that $R$ is Gorenstein if and only if

$$
\mathscr{E} x t_{R}^{i}(R / M, R)= \begin{cases}R / M & \text { for } i=\operatorname{dim} R \\ 0 & \text { for } i \neq \operatorname{dim} R .\end{cases}
$$

If $R$ is Gorenstein we have $\mathscr{H}_{M}^{d}(R)=\mathscr{E}_{R}(k)(n)$ for some $n \in Z$. Hence $\mathscr{K}_{\hat{R}}=\hat{R}(-n)$ by Proposition (1.3). By the uniqueness of canonical modules we have $\mathscr{K}_{R}=R(-n)$. Conversely assume that $\mathscr{K}_{R}=R(-n)$ for some $n \in Z$. By Proposition (1.8) we get

$$
\mathscr{E} x t_{\hat{R}}^{i}(\hat{R} / \hat{M}, \hat{R})=\mathscr{H}_{o m_{\hat{R}}}\left(\mathscr{H}_{\hat{M}}^{d_{-i}}\left(\hat{R} / \hat{M}, \mathscr{E}_{\hat{R}}(k)\right)(n)\right.
$$

for all $i \geq 0$, where $\hat{M}$ is the maximal homogeneous ideal of $\hat{R}$. Hence $\hat{R}$ is Gorenstein since $\mathscr{H}_{M}^{0}(\hat{R} / \hat{M})=\hat{R} / \hat{M}$ and $\mathscr{H}_{\hat{M}}^{i}(\hat{R} / \hat{M})=0$ for $i>0$. Since $\hat{R}$ is faithfully flat over $R$ it follows that $R$ is Gorenstein.

Remark. Let $a=\max \left\{n \mid \mathscr{H}_{M}^{d}(R)_{n} \neq 0\right\}$. If $R$ is Gorenstein we have $\mathscr{K}_{R}=R(a)$. In the sequel we denote this number by $a(R)$ and call it the $a$-invariant of $R$. 
Proposition (1.10). Let $R \rightarrow S$ be a finite homomorphism of graded rings defined over local rings. Assume that $R$ is Cohen-Macaulay and has a canonical module. Then

$$
\mathscr{K}_{S}=\mathscr{E} x t_{R}^{r}\left(S, \mathscr{K}_{R}\right),
$$

where $r=\operatorname{dim} R-\operatorname{dim} S$.

Proof. Let $n_{0}$ be the maximal ideal of $S_{0}$ and $\hat{S}_{0}$ be the $n_{0}$-adic completion of $S_{0}$. Since $S_{0}$ is finite over $R_{0}$ we have $\hat{S}_{0}=S_{0} \otimes_{R_{0}} \hat{R}_{0}$. Let $N$ be the maximal homogeneous ideal of $S$ and $\hat{N}=N \otimes_{R_{0}} \hat{R}_{0}$. Let $\hat{S}=$ $S \otimes_{R_{0}} \hat{R}_{0}$. Note that $\mathscr{H}_{o m_{\hat{R}}}\left(\hat{S}, \mathscr{E}_{\hat{R}}(k)\right)$ is the injective envelope of $\hat{S} / \hat{N}$ in $M_{H}(\hat{S})$.

$$
\begin{aligned}
\mathscr{K}_{\hat{S}} & =\mathscr{H}_{o m_{\hat{S}}}\left(\mathscr{H}_{\hat{\hat{N}}}^{s}(\hat{S}), \mathscr{E}_{\hat{S}}(\hat{S} / \hat{N})\right) \quad(s=\operatorname{dim} S) \\
& =\mathscr{H}_{o_{\hat{S}}}\left(\mathscr{H}_{M}^{s}(\hat{S}), \mathscr{H}_{o m_{\hat{R}}}\left(\hat{S}, \mathscr{E}_{\hat{R}}(k)\right)\right) \\
& =\mathscr{H}_{o m_{\hat{R}}}\left(\mathscr{H}_{\hat{M}}^{s}(\hat{S}), \mathscr{E}_{\hat{R}}(k)\right) \\
& =\mathscr{E}_{x t \hat{R}}\left(\hat{S}, \mathscr{K}_{\hat{R}}\right) \\
& =\mathscr{E}_{x t r}\left(S, \mathscr{K}_{R}\right) \otimes_{R} \hat{R} .
\end{aligned}
$$

Since $S$ is finite over $R$ it follows that $\mathscr{K}_{S}=\mathscr{E} x t_{R}^{r}\left(S, \mathscr{K}_{R}\right)$.

Corollary (1.11). If moreover $R$ is Gorenstein in Proposition (1.10) we get $\mathscr{K}_{S}=\mathscr{E} x t_{R}^{r}(S, R)(n)$ for some $n \in Z$.

From Corollary (1.11) one knows that for any $p \in \operatorname{Supp}\left(\mathscr{K}_{S}\right)\left(\mathscr{K}_{s}\right)_{p}$ is a canonical module of the local ring $S_{p}$ in the sense of [HK].

\section{§ 2. Preliminaries}

In this section we collect fundamental facts about the local cohomology of Rees algebras over Noetherian local rings.

Let $(A, m, k)$ be a local ring and $I$ an ideal of $A$. We put $R(I)=$ $\oplus_{n \geq 0} I^{n}$ and call this graded $A$-algebra the Rees algebra of $I$. Let $I=$ $\left(a_{1}, \cdots, a_{n}\right)$. Then $R(I)$ can be identified with the subalgebra $A\left[a_{1} X, \cdots\right.$, $\left.a_{n} X\right]$ of the polynomial ring $A[X]$ in one variable. Throughout this paper we use this identification without mentioning. Let $M=m R(I)+$ $\left(a_{1} X, \cdots, a_{n} X\right) R(I)$ be the maximal homogeneous ideal of $R(I)$. Let $G(I)$ $=\oplus_{n \geq 0} I^{n} / I^{n+1}$ be the associated graded ring of $I$. Note that

$$
G(I)=R(I) / I R(I) \quad \text { and } \quad A=R(I) / R(I)_{+},
$$

where $R(I)_{+}=\oplus_{n>0} I^{n}$. Let $\ell(I)=\operatorname{dim} R(I) / m R(I)$; we call this number the analytic spread of $I$. The analytic spread $\ell(I)$ of $I$ is equal to the 
minimum number of generators of a minimal reduction of $I$ if the residue field $k$ is infinite (cf. [NR]).

Proposition (2.1). Let $(A, m, k)$ be a local ring and $I$ an ideal of $A$ with ht $(I)>0$. If $R(I)$ is Cohen-Macaulay then

a) $a(G(I))<0$ and

b) for $i<\operatorname{dim} A$ we have

$$
\mathscr{H}_{M}^{i}(G(I))_{n}= \begin{cases}H_{m}^{i}(A) & \text { for } n=-1 \\ 0 & \text { for } n \neq-1 .\end{cases}
$$

Proof. For b) see the proof of $[\mathrm{HI}]$, Proposition (1.5). Let $J=R(I)_{+}$. From the exact sequences

and

$$
0 \longrightarrow J \longrightarrow R(I) \longrightarrow A \longrightarrow 0
$$

$$
0 \longrightarrow J(1) \longrightarrow R(I) \longrightarrow G(I) \longrightarrow 0
$$

we obtain the exact sequences of local cohomology

$$
0 \longrightarrow H_{m}^{d}(A) \longrightarrow \mathscr{H}_{M}^{d+1}(J) \stackrel{f}{\longrightarrow} \mathscr{H}_{M}^{d+1}(R(I)) \longrightarrow 0
$$

and

$$
0 \longrightarrow \mathscr{H}_{M}^{d}(G(I)) \longrightarrow \mathscr{H}_{M}^{d+1}(J)(1) \stackrel{g}{\longrightarrow} \mathscr{H}_{M}^{d+1}(R(I)) \longrightarrow 0,
$$

where $d=\operatorname{dim} A$. From this one gets the isomorphisms

$$
f_{n}: \mathscr{H}_{M}^{d+1}(J)_{n} \longrightarrow \mathscr{H}_{M}^{d+1}(R(I))_{n} \quad \text { for } n \neq 0
$$

and surjective homomorphisms

$$
g_{n}: \mathscr{H}_{M}^{d+1}(J)_{n} \longrightarrow \mathscr{H}_{M}^{d+1}(R(I))_{n-1} \quad \text { for all } n .
$$

Since $\mathscr{H}_{M}^{d+1}(J)$ and $\mathscr{H}_{M}^{d+1}(R(I))$ are Artinian $R(I)$-modules their homogeneous components of sufficiently large degree are zero. By an easy diagram chase we know that $\mathscr{H}_{M}^{d+1}(J)_{n}=0$ for $n \geq 1$ and $\mathscr{H}_{M}^{d+1}(R(I))_{n}=0$ for $n \geq 0$. Now it is easy to see that $a(G(I))<0$.

Corollary (2.2). Let $A$ and $I$ be the same as in Proposition (2.2). Then $\mathscr{H}_{M}^{d+1}(R(I))_{n}=0$ for $n \geq 0$.

Proof. This follows from the proof of Proposition (2.1).

If, inparticular, $I=m$ we have the following result.

Proposition (2.3). If $d=\operatorname{dim} A>0$ the following conditions are 
equivalent.

(1) $R(m)$ is Cohen-Macaulay.

(2) a) $a(G(m))<0$ and

b) for $i<d$ we have

$$
\mathscr{H}_{M}^{i}(G(m))_{n}= \begin{cases}H_{m}^{i}(A) & \text { for } n=-1 \\ 0 & \text { for } n \neq-1 .\end{cases}
$$

In this case $A$ and $G(m)$ are Buchsbaum.

Proof. See $\left[I_{1}\right]$.

For the technical simplicity in the rest of this paper we assume that every local ring has an infinite residue field.

Lemma (2.4). Let $A$ and $I$ be the same as above and let $q$ be a minimal reduction of $I$. We put $r(q)=\min \left\{r \in Z \mid I^{r+1}=q I^{r}\right\}$. If $\operatorname{ht}(I)=\ell(I)$ we have $r(q) \geq a(G(I))+\operatorname{ht}(I)$.

Proof. See [HI], Lemma (2.3).

Lemma (2.5). Let $A$ and $I$ be the same as above. We put

$$
n_{i}=\max \left\{n \in Z \mid \mathscr{H}_{M}^{i}(G(I))_{n} \neq 0\right\} \quad \text { for } 0 \leq i \leq d=\operatorname{dim} A .
$$

If $I$ is m-primary we have $r(q) \leq \max _{i}\left\{n_{i}+i\right\}$ for any minimal reduction $q$ of $I$.

Proof. Let $x \in A$. We denote by $x^{*}$ the initial form of $x$ with respect to $-I$. Let $q=\left(a_{1}, \cdots, a_{d}\right)$ and $q^{*}=\left(a_{1}^{*}, \cdots, a_{d}^{*}\right)$. Then

$$
r(q)=\max \left\{r \in Z \mid\left(G(I) / q^{*}\right)_{r} \neq 0\right\} .
$$

If $\operatorname{dim} G(I)=0$ the assertion is clear. Let $\operatorname{dim} G(I)>0$. Since the residue field is infinite we may assume that $l_{G(I)}\left(\left(0: a_{1}^{*}\right)\right)<\infty$. From the exact sequences

$$
0 \longrightarrow G(I) /\left(0: a_{1}^{*}\right)(-1) \longrightarrow G(I) \longrightarrow G(I) / a_{1}^{*} G(I) \longrightarrow 0
$$

and

$$
0 \longrightarrow\left(0: a_{1}^{*}\right) \longrightarrow G(I) \longrightarrow G(I) /\left(0: a_{1}^{*}\right) \longrightarrow 0
$$

we get the exact sequence

$$
\mathscr{H}_{M}^{i}(G(I)) \longrightarrow \mathscr{H}_{M}^{i}\left(G(I) / a_{1}^{*} G(I)\right) \longrightarrow \mathscr{H}_{M}^{i+1}(G(I))(-1)
$$

for $0 \leq i \leq d$. Let $n_{i}^{\prime}=\max \left\{n \in Z \mid \mathscr{H}_{M}^{i}\left(G(I) / a_{1}^{*} G(I)\right)_{n} \neq 0\right\}$. Then $n_{i}^{\prime} \leq$ $\max \left\{n_{i}, n_{i+1}+1\right\}$. By induction we have $r(q) \leq \max \left\{n_{i}+i\right\}$. 


\section{§3. The Gorensteinness of Rees algebras}

This section is devoted to the proof of the following theorem.

Theorem (3.1). Let $(A, m, k)$ be a local ring and $I$ an ideal of $A$. Suppose that $R(I)$ is Cohen-Macaulay and grade $(I) \geq 2$. Then the following conditions are equivalent.

(1) $R(I)$ is Gorenstein.

(2) $K_{A}=A$ and $\mathscr{K}_{G(I)}=G(I)(-2)$.

Remark. Since $A$ and $G(I)$ are homomorphic images of $R(I), A$ and $G(I)$ have canonical modules if $R(I)$ is Gorenstein.

We need several preliminaries to prove this theorem.

Lemma (3.2). Let $A$ be a local ring which has a canonical module $K_{A}$. Then the following conditions are equivalent.

(1) A satisfies $\left(S_{2}\right)$.

(2) A satisfies $\left(S_{2}\right)$.

(3) $\operatorname{Hom}_{A}\left(K_{A}, K_{A}\right)=A$.

Proof. See [A], (4.4) and (4.5).

Lemma (3.3). Let $A$ and $I$ be the same as in Theorem (3.1). Let $a \in$ $I-I^{2}$ be an element whose initial form in $G(I)$ is a non zero-divisor. We put $\bar{R}=R(I) /(a, a X)$. If $R(I)$ is Gorenstein and grade $(I) \geq 2$ we have $\mathscr{H}_{M}^{d-1}(\bar{R})=0$, where $\operatorname{dim} A=d$.

Proof. Let $R=R(I)$ and $G=G(I)$. Since $a$ is a non zero divisor, by Propositions (1.8) and (1.9), it is enough to show that $\mathscr{E}_{x t_{R / a}^{1} R}^{1}(\bar{R}, R / a R)$ $=0$. Let $I=\left(a_{1}, \cdots, a_{n}\right)$. Then we have the exact sequence

$$
(R / a R)^{n}(-1) \underset{\left[\begin{array}{c}
a_{1} \\
\vdots \\
a_{n}
\end{array}\right]}{\longrightarrow} R / a R(-1) \underset{a X}{\longrightarrow} R / a R \longrightarrow 0
$$

Applying the functor $\mathscr{H}_{o m_{R / a}}(, R / a R)$ to this sequence, we see

$$
\mathscr{E} x t_{R / a R}^{1}(\bar{R}, R / a R)=(a R: I R) /(a, a X) .
$$

Let $f^{m} \in(a R: I R)$, where $f \in I^{m}$ and $m \geq 0$. Then we have

$$
f \in(a A: I) \cap\left(I^{m+1}: I\right) \subset(a A: I) \cap\left(I^{m+1}: a\right) .
$$

Since grade $(I) \geq 2$ we have $(a A: I)=a A$. That $a^{*}$ is a non zero-divisor of $G(I)$ is equivalent to that $\left(I^{m}: a\right)=I^{m-1}$ for all $m>0$. Hence we 
have $\left(I^{m+1}: a\right)=I^{m}$. Therefore $f \in I^{m} \cap a A=a I^{m-1}$. This means $(a R: I R)$ $=(a, a X)$, which completes the proof.

Lemma (3.4). Let $A$ and $I$ be the same as in Theorem (3.1). Assume that $R(I)$ is Cohen-Macaulay and $\mathscr{K}_{G(I)}=G(I)(-2)$. Then

$$
\mathscr{H}_{\mathrm{om}_{R(I)}}\left(k, \mathscr{H}_{M}^{d+1}(R(I))\right)_{n}=0 \quad \text { for } n \neq-1,
$$

where $d=\operatorname{dim} A$ and $k=R(I) / M$.

Proof. Let $R$ and $G$ be as in the proof of Lemma (3.3). Put $J=$ $\oplus_{n>0} R_{n}$. Then we get the exact sequence (cf. the proof of Proposition (2.1))

(I) $\quad 0 \longrightarrow \mathscr{H}_{o m_{R}}\left(k, H_{m}^{d}(A)\right) \longrightarrow \mathscr{H}_{o m_{R}}\left(k, \mathscr{H}_{M}^{d+1}(J)\right) \stackrel{f}{\longrightarrow} \mathscr{H}_{o m_{R}}\left(k, \mathscr{H}_{M}^{d+1}(R)\right)$

$$
\longrightarrow \mathscr{E} x t_{R}^{1}\left(k, H_{m}^{d}(A)\right)
$$

and

(II) $0 \longrightarrow \mathscr{H}_{o m_{R}}\left(k, \mathscr{H}_{M}(G)\right) \longrightarrow \mathscr{H}_{o m_{R}}\left(k, \mathscr{H}_{M}^{d+1}(J)\right)(1) \stackrel{g}{\longrightarrow} \mathscr{H}_{o m_{R}}\left(k, \mathscr{H}_{M}^{d+1}(R)\right)$

$$
\longrightarrow \mathscr{E}^{x} t_{R}^{1}\left(k, \mathscr{H}_{M}^{d}(G)\right) \text {. }
$$

Since $\mathscr{H}_{\mathrm{om}_{R}}\left(k, H_{m}^{d}(A)\right)$ is concentrated in degree 0 and since $\mathscr{E} x t_{R}^{1}\left(k, H_{m}^{d}(A)\right)_{n}$ $=0$ for $n \leq-2$ from (I) we get isomorphisms

$$
f_{n}: \mathscr{H}_{o m_{R}}\left(k, \mathscr{H}_{M}^{d+1}(J)\right)_{n} \longrightarrow \mathscr{H}_{o m_{R}}\left(k, \mathscr{H}_{M}^{d+1}(R)\right)_{n}
$$

for $n \leq-2$. By assumption $\mathscr{H}_{\mathrm{om}_{R}}\left(k, \mathscr{H}_{M}^{d}(G)\right)_{n}=0$ for $n \neq-2$. (II) yields injective homomorphisms

$$
g_{n}: \mathscr{H}_{o m_{R}}\left(k, \mathscr{H}_{M}^{d+1}(J)\right)_{n} \longrightarrow \mathscr{H}_{o m_{R}}\left(k, \mathscr{H}_{M}^{d+1}(R)\right)_{n-1}
$$

for $n \leq-2$. Since $\mathscr{H}_{M}^{d+1}(J)$ and $\mathscr{H}_{M}^{d+1}(R)$ are Artinian

$$
\mathscr{H}_{o m_{R}}\left(k, \mathscr{H}_{M}^{d+\mathrm{r}}(J)\right)_{n}=\mathscr{H}_{o m_{R}}\left(k, \mathscr{H}_{M}^{d+1}(R)\right)_{n}=0 \quad \text { for } n \ll 0 .
$$

Now, it is easy to see that

$$
\mathscr{H}_{o m_{R}}\left(k, \mathscr{H}_{M}^{d+1}(R)\right)_{n}=0
$$

for $n \leq-2$. On the other hand, by Corollary (2.2) we have $\mathscr{H}_{M}^{d+1}(R)_{n}=0$ for $n \geq 0$. This completes the proof.

Lemma (3.5). Let $(A, m, k)$ be a local ring and $I$ an ideal of $A$ such that $R(I)$ is Cohen-Macaulay. Suppose that grade $(I) \geq n>0$, Then $A$ and $G(I)$ satisfy $\left(S_{n}\right)$. 
Proof. We may assume that $A$ is complete. Let $G=G(I)$. Let $B$ be a Gorenstein local ring such that $A$ is a homomorphic image of $B$ and $d=\operatorname{dim} A=\operatorname{dim} B$. Let $n$ be the maximal ideal of $B$. By the local duality we have

$$
\operatorname{Ext}_{B}^{i}(A, B)=\operatorname{Hom}_{B}\left(H_{n}^{d-i}(A), E_{B}(B / n)\right) \quad \text { for } i \geq 0,
$$

where $E_{B}(B / n)$ is the injective envelope of $B / n$ as $B$-module. By Proposition (2.1) we see that $\operatorname{Ext}_{B}^{i}(A, B)$ is annihilated by $I$ for $i>0$. Let $p \in$ $\operatorname{Spec}(A)$ and $P$ be the inverse image of $p$ in $B$. Then if $p \not \supset I$ we have

$$
\operatorname{Ext}_{B_{P}}^{i}\left(A_{p}, B_{P}\right)=0 \quad \text { for } i>0 \text {. }
$$

Hence $A_{p}$ is Cohen-Macaulay. If $p \supset I$ we have $\operatorname{depth} A_{p} \geq n$ by assumption. Therefore $A$ satisfies $\left(S_{n}\right)$.

To prove the assertion on $G$ we use induction on $\operatorname{dim} A / I$. Let $\operatorname{dim} A / I=0$. By Proposition (2.1) we know that $l_{G}\left(\mathscr{H}_{M}^{i}(G)\right)<\infty$ for $i<d$ and depth $G_{N} \geq n$, where $N$ is the maximal homogeneous ideal of $G$. Hence $G$ satisfies $\left(S_{n}\right)$ because $G_{Q}$ is Cohen-Macaulay for $Q \in \operatorname{Spec}(G)$ $-\{N\}$. Let $\operatorname{dim} A / I>0$. Note that $G$ can be written as a homomorphic image of a Gorenstein graded ring of the same dimension. By Proposition (1.8) we see that $G_{p}$ is Cohen-Macaulay if $p \not \supset G_{+}$, where $G_{+}=\oplus_{n>0} G_{n}$. Assume that $p \supset G_{+}$and $p \neq N$. Then $p \cap A / I=P / I$ for some $P \in \operatorname{Spec}(A)$ - $\{m\}$. Since $R(I)_{P}$ is Cohen-Macaulay and $\operatorname{dim} A / I>\operatorname{dim} A_{P} / I A_{P}$ one knows that $G_{P}$ satisfies $\left(S_{n}\right)$ by induction on $\operatorname{dim} A / I$.

Proof of Theorem (3.1). First we show that if $h t(I)>0$ and $R(I)$ is Cohen-Macaulay then there is an element $a \in I-I^{2}$ whose initial form in $G(I)$ is a non zero-divisor. Since $h t(I R(I))>0$ one can choose an element $b \in I$ which is a non zero-divisor on $R(I)$. Noting that $R(I) / I R(I)$ $+I X R(I)=A / I$, we have $h t(I R(I)+I X R(I))=\operatorname{dim} R(I)-\operatorname{dim} A / I=d+$ $1-\operatorname{dim} A / I \geq 2$. Since the residue field of $A$ is infinite we can choose an element $c+a X$ of $I R(I)+I X R(I)$ such that $b, c+a X$ is an $R(I)$-sequence and $a \in I-I^{2}$. Since $b$ is also a non zero-divisor on $A$ one can easily verify that $(b R(I): b X)=I R(I)$. This implies that there exists an exact sequence

$$
0 \longrightarrow G(I)(-1) \longrightarrow R(I) / b R(I) \longrightarrow R(I) /(b, b X) R(I) \longrightarrow 0 .
$$

By the choice of $c+a X$ we see that $c+a X$ is a non zero-divisor on $G(I)$. The canonical image of $c+a X$ in $G(I)=R(I) / I R(I)$ is nothing but 
the initial form of $a$ because $c \in I$. Therefore the initial form $a^{*}$ of $a$ in $G(I)$ is a non zero-divisor on $G(I)$.

(1) $\Rightarrow(2)$ : Let $R=R(I)$ and $G=G(I)$. Let $a \in I-I^{2}$ be as above. Since $a$ is a non zero-divisor on $A$ there are two exact sequences

$$
0 \longrightarrow A \longrightarrow R / a X R \longrightarrow R /(a, a x) \longrightarrow 0
$$

and

$$
0 \longrightarrow G(-1) \longrightarrow R / a R \longrightarrow R /(a, a X) \longrightarrow 0 \text {. }
$$

These exact sequences induce the exact sequences by Lemma (3.3)

$$
0 \longrightarrow \mathscr{H}_{M}^{d}(A) \longrightarrow \mathscr{H}_{M}^{d}(R / a X R) \longrightarrow \mathscr{H}_{M}^{d}(R /(a, a X)) \longrightarrow 0
$$

and

$(++) \quad 0 \longrightarrow \mathscr{H}_{M}^{d}(G)(-1) \longrightarrow \mathscr{H}_{M}^{d}(R / a R) \longrightarrow \mathscr{H}_{M}^{d}(R /(a, a X)) \longrightarrow 0$,

where $d=\operatorname{dim} A$ and $M$ is the maximal homogeneous ideal of $R$ as before. Since $R$ is Gorenstein $\mathscr{K}_{R}=R(n)$ for some $n \in Z$. Since $a X$ is a non zero divisor of degree 1 we have $\mathscr{K}_{R / a X R}=R / a X R(n+1)$. From the exact sequence ( + ) we know that $n=-1$ and $K_{A}=A / J$ for some ideal $J$ of $A$. From $(++)$ we have $\mathscr{K}_{G}=G / L(-2)$ for some homogeneous ideal $L$ of $G$. By Lemma (3.5) $A$ and $G$ satisfy $\left(S_{2}\right)$, hence by Lemma (3.2) we have $J=0$ and $L=0$.

$(2) \Rightarrow(1)$ : From the exact sequences $(\sharp)$ and $(\# \#)$ we get two injections $\mathscr{H}_{M}^{d-1}(R /(a, a X)) \rightarrow \mathscr{H}_{M}^{d}(A)$ and $\mathscr{H}_{M}^{d-1}(R /(a, a X)) \rightarrow \mathscr{H}_{M}^{d}(G)(-1)$ since $R$ is Cohen-Macaulay. From the first one we know that $\mathscr{H}_{M}^{d-1}(R /(a, a X))$ is concentrated in degree 0 . The assumption $\mathscr{K}_{G}=G(-2)$ shows that $\mathscr{H}_{M}^{d}(G)_{n}$ $=0$ for $n \geq-1$. From the second injection we see that $\mathscr{H}_{M}^{d-1}(R /(a, a X))$ $=0$. Hence we have the exact sequences $(+)$ and $(++)$. By Lemma (3.4) we know that $\mathscr{H}_{o m_{R}}\left(k, \mathscr{H}_{M}^{d}(R / a X R)\right)$ is concentrated in degree 0. By (+) we get

$$
\mathscr{H}_{\mathrm{om}_{R}}\left(k, \mathscr{H}_{M}^{d}(R / a X R)\right)=\operatorname{Hom}_{A}\left(k, H_{m}^{d}(A)\right)
$$

since $\mathscr{H}_{M}^{d}(R / a R)_{n}=\mathscr{H}_{M}^{d}(R /(a, a X))_{n}=0$ for $n \geq 0$ by Corollary (2.2). By the assumption $K_{A}=A$ we have $\operatorname{Hom}_{A}\left(k, H_{m}^{l}(A)\right)=k$. This shows that $R$ is Gorenstein.

Let $I$ be an ideal of a local ring and $q$ a minimal reduction of $I$. We put $r(q)=\min \left\{r \mid I^{r+1}=q I^{r}\right\}$. We call $r(q)$ the reduction exponent of $q$. 
Corollary (3.6) Let $A$ be a local ring and $I$ an ideal of $A$ such that ht $(I)=\ell(I)>0$ and $R(I)$ is Cohen-Macaulay. Then we have:

(1) Suppose that $a(G(I)) \geq-2$. Then we have $r(q)=\operatorname{ht}(I)-1$ or ht $(I)-2$ for any minimal reduction $q$ of $I$.

(2) Suppose moreover that grade $(I) \geq 2$ and $R(I)$ is Gorenstein. Then for any minimal reduction $q$ of $I$ we have $r(q)=\mathrm{ht}(I)-2$ if and only if $\operatorname{depth} A \geq \operatorname{dim} A / I+2$.

Proof. (1) By induction on $\operatorname{dim} A / I$. If $\operatorname{dim} A / I=0$ this follows from Lemmas (2.4) and (2.5). Let $\operatorname{dim} A / I>0$. Choose an element $b \in A$ whose image in $A / I$ is a part of system of parameters of $A / I$. By Proposition (1.5) $b$ is a non zero-divisor on $G(I)$ and $R(I)$ and we have $R(I) / b R(I)$ $=R(I(A / b A))$ and $G(I)=G(I(A / b A))$. It is easy to see that the ideal $I(A / b A)$ in $A / b A$ satisfies the same assumption on $I$. By induction hypothesis we have $r(q(A / b A))=$ ht $(I)-1$ or ht $(I)-2$. By Nakayama's lemma we have $r(q(A / b A))=r(q)$.

(2) First we assume that $\operatorname{depth} A \geq \operatorname{dim} A / I+2$. If $\operatorname{dim} A / I=0$ we have $r(q)=h t(I)-2$ by Proposition (2.1), Lemmas (2.4) and (2.5). We proceed by induction on $\operatorname{dim} A / I$. Let $\operatorname{dim} A / I>0$. Then by assumption depth $A \geq 3$. Let $a_{1}, a_{2}$ be a regular sequence in $I$. One can choose an element $b \in m$ so that $a_{1}, a_{2}, b$ is a regular sequence and the image of $b$ in $A / I$ is a part of system of parameters of $A / I$. Then grade $(I(A / b A))$ $\geq 2$ and $R(I(A / b A))$ is Gorenstein. Since $\operatorname{depth} A / b A \geq \operatorname{dim} A /(b, I)+2$ we have $r(q)=\mathrm{ht}(I)-2$ by induction hypothesis.

Conversely assume that $r(q)=\mathrm{ht}(I)-2$. Let $b_{1}, \cdots, b_{s} \in m$ be a system of parameters of $A / I$. We set $\bar{A}=A /\left(b_{1}, \cdots, b_{s}\right)$. Since $b_{1}, \cdots, b_{s}$ is a regular sequence we have only to show that $\operatorname{depth} \bar{A} \geq 2$. Let $\bar{I}=$ $I \bar{A}$ and $\bar{q}=q \bar{A}$. Since $r(\bar{q})=h t(\bar{I})-2$ we see that $\mathscr{H}_{M}^{h}(G(\bar{I}))_{n}=0$ for $n \geq-1$ by Lemma (2.4), where $h=\mathrm{ht}(I)$. By [HI], Proposition (1.5) we know that $b_{1}, \cdots, b_{s}$ is a $G(I)$-sequence. Let $q_{i}=\left(b_{1}, \cdots, b_{i}\right)$ for $1 \leq i \leq s$. Then we see that $G(I) / q_{i} G(I)=G\left(I\left(A / q_{i}\right)\right)$. We set $G_{i}=G(I) / q_{i} G(I)$ for $1 \leq i \leq s$. Then we have an exact sequence

$$
\mathscr{H}_{M}^{d-i}\left(G_{i-1}\right) \stackrel{b_{i}}{\longrightarrow} \mathscr{H}_{M}^{d-i}\left(G_{i-1}\right) \longrightarrow \mathscr{H}_{M}^{d-i}\left(G_{i}\right) \longrightarrow \mathscr{H}_{M}^{d-i+1}\left(G_{i-1}\right) .
$$

Since $r\left(q\left(A / q_{i}\right)\right)=\operatorname{ht}(I)-2$ we know that $\mathscr{H}_{M}^{d-i}\left(G_{i}\right)_{n}=0$ for $n \geq-1$ by Lemma (2.4). By Proposition (2.1) we see that $H_{m}^{d-i}\left(A / q_{i-1}\right)=b_{i} H_{m}^{d-i}\left(A / q_{i-1}\right)$ for $1 \leq i \leq s$. 
This implies that $K_{A / q_{i}}=A / q_{i}$ for $0 \leq i \leq s$, where $q_{0}=0$. In particular, $K_{\bar{A}}=\bar{A}$. One sees that $\operatorname{depth} \bar{A} \geq 2$ by [A]. The following is a generalization of a result in [GS].

Corollary (3.7). Let $A$ be a Cohen-Macaulay local ring and $I$ an ideal of $A$ with $\mathrm{ht}(I)=\ell(I) \geq 2$. Then the following conditions are equivalent.

(1) $R(I)$ is Gorenstein.

(2) $G(I)$ is Gorenstein and $a(G(I))=-2$.

(3) $G(I)$ is Gorenstein and there exists a minimal reduction $q$ of $I$ such that $r(q)=\mathrm{ht}(I)-2$.

In this case $A$ is Gorenstein.

Proof. This follows from Theorem (3.1), Corollary (3.6) and the fact that the Gorensteinness of $G(I)$ implies that of $A$.

Corollary (3.8). Let $A$ and $I$ be the same as in Corollary (3.6). Suppose that

(1) $R(I)$ is Gorenstein,

(2) $l_{A}\left(H_{m}^{i}(A)\right)<\infty$ for $i<d=\operatorname{dim} A$ and

(3) $2 \mathrm{ht}(I) \leq \operatorname{dim} A$.

Then $A$ is Gorenstein.

Proof. By Corollary (3.7) it is sufficient to prove that $A$ is CohenMacaulay. Let $b_{1}, \cdots, b_{s}$ be a system of parameters of $A / I$. We put $q_{i}=\left(b_{1}, \cdots, b_{i}\right)$ and $G_{i}=G(I) / q_{i} G(I)$ for $1 \leq i \leq s$. Let $a_{1}, \cdots, a_{h}, h=$ ht $(I)$, be a minimal generators of a minimal reduction of $I$. Then $a_{1}, \cdots, a_{h}, b_{1}, \cdots, b_{s}$ is a system of parameters of $A$. Since $l_{A}\left(H_{m}^{i}(A)\right)<\infty$ for $i<d$ we know that if $t \leq \operatorname{depth} A$ then any $t$ elements of a system of parameters of $A$ form a regular sequence by [CST], (3.3). Hence we have grade $\left(I\left(A / q_{i}\right)\right) \geq 2$ for $1 \leq i<s$ by [HI], Proposition (1.5). We are going to show that $H_{m}^{d-s+i}\left(A / q_{s-j}\right)=0$ for $2 \leq j \leq s-2$ and $1 \leq i \leq j-1$ by induction on $j$. Let $j=2$. From the exact sequence

$$
\mathscr{H}_{M}^{d-s+1}\left(G_{s-2}\right) \stackrel{b_{s-1}}{\longrightarrow} \mathscr{H}_{M}^{d-s+1}\left(G_{s-2}\right) \longrightarrow \mathscr{H}_{M}^{d-s+1}\left(G_{s-1}\right)
$$

we get $H_{m}^{d-s+1}\left(A / q_{s-2}\right)=b_{s-1} H_{m}^{d-s+1}\left(A / q_{s-2}\right)$ by Theorem (3.1) and Proposition (2.1) since $R\left(I\left(A / q_{s-1}\right)\right)$ is Gorenstein. By the assumption (2) we get $H_{m}^{d-s+1}\left(A / q_{s-2}\right)=0$. Let us assume that our assertion is true for $j<s-2$ and we will prove that the assertion is true for $j+1$. Since $b_{s-j}$ is a 
non zero-divisor on $A / q_{s-j-i}$ we obtain the exact sequence

$$
H_{m}^{d-s+i}\left(A / q_{s-j-1}\right) \stackrel{b_{s-j}}{\longrightarrow} H_{m}^{d-s+i}\left(A / q_{s-j-1}\right) \longrightarrow H_{m}^{d-s+i}\left(A / q_{s-j}\right)
$$

for $i>0$. By the induction hypothesis $H_{m}^{d-s+i}\left(A / q_{s-j}\right)=0$ for $1 \leq i \leq j-1$. Therefore $H_{m}^{d-s+i}\left(A / q_{s-j-1}\right)=0$ for $1 \leq i \leq j-1$ by assumption (2). It remains to prove that $H_{m}^{d-s+j}\left(A / q_{s-j-1}\right)=0$. But this can be proved by the same method used for $j=2$. Hence, in particular, we get $H_{m}^{i}(A)=0$ for $h+1 \leq i \leq d$. By assumption (3) we get $\operatorname{depth} A \geq \operatorname{dim} A / I+1 \geq h$ +1 cf. [HI]. Therefore $A$ is Cohen-Macaulay.

\section{§4. Example}

In this section we construct a local ring $(A, m, k)$ such that $R(m)$ is Gorenstein but $A$ is not Cohen-Macaulay. For a local ring $A$ we denote the multiplicity of $A$ by $e(A)$.

Lemma (4.1). Let $(A, m, k)$ be a local ring with $\operatorname{dim} A=3$ and $q=$ $\left(a_{1}, a_{2}, a_{3}\right)$ be a minimal reduction of $m$. Let

$$
I=\left(\left(a_{1}, a_{2}\right): a_{3}\right)+\left(\left(a_{2}, a_{3}\right): a_{1}\right)+\left(\left(a_{1}, a_{3}\right): a_{2}\right)+m^{2} .
$$

Then $R(m)$ is Cohen-Macaulay if and only if $m^{3}=q m^{2}$ and $l_{A}\left(I / m^{2}\right)=$ $3\left(l_{A}(A / q)-e(A)\right)+3$.

Proof. See $\left[I_{2}\right]$, Theorem 5.

Lemma (4.2). Let $A$ be the same as in Lemma (4.1). Suppose that $R(m)$ is Cohen-Macaulay and $A$ is not Cohen-Macaulay. If $l_{A}\left(m / m^{2}\right)=6$ we have

(1) $A$ is a Buchsbaum ring with depth $A=2$ and $l_{A}\left(H_{m}^{2}(A)\right)=1$,

(2) $m^{2}=q m$ for any minimal reduction $q$ of $m$ and

(3) $e(A)=3$.

Proof. See $\left[\mathrm{I}_{2}\right]$, Corollary 11.

Example (1). Let $k$ be a field and $X_{i}, Y_{i}(1 \leq i \leq 3)$ be indeterminates over $k$. We put

$$
A=k\left[\left[X_{1}, X_{2}, X_{3}, Y_{1}, Y_{2}, Y_{3}\right]\right] /\left(X_{1} Y_{1}+X_{2} Y_{2}+X_{3} Y_{3},\left(Y_{1}, Y_{2}, Y_{3}\right)^{2}\right) .
$$

Then $A$ is not Cohen-Macaulay but $R(m)$ is Cohen-Macaulay. By Lemma (4.2) $e(A)=3$ (cf. $\left[\mathrm{I}_{1}\right]$ and $\left.\left[\mathrm{I}_{2}\right]\right)$. 
Example (2). Let $k$ be a field of $\operatorname{ch}(k)=2$ and $X_{1}, X_{2}, X_{3}, Y_{1}, Y_{2}, Y_{3}, Y_{4}$ indeterminates over $k$. Let

$$
\begin{aligned}
A & =k\left[\left[X_{1}, X_{2}, X_{3}, Y_{1}, Y_{2}, Y_{3}, Y_{4}\right]\right] / J \\
& =k\left[\left[x_{1}, x_{2}, x_{3}, y_{1}, \cdots, y_{4}\right]\right]
\end{aligned}
$$

where $J$ is the ideal generated by $X_{1} Y_{1}+X_{2} Y_{2}+X_{3} Y_{3}, Y_{1}^{2}, Y_{2}^{2}, Y_{3}^{2}, Y_{4}^{2}, Y_{1} Y_{4}$, $Y_{2} Y_{4}, Y_{3} Y_{4}, Y_{1} Y_{2}-X_{3} Y_{4}, Y_{2} Y_{3}-X_{1} Y_{4}$ and $Y_{1} Y_{3}-X_{2} Y_{4}$.

Then $A$ is not Cohen-Macaulay but $R(m)$ is Gorenstein.

To prove this we need the following lemma.

Lemma (4.3). $\quad\left(0: y_{4}\right)=\left(y_{1}, \cdots, y_{4}\right)$

Proof. Let $R=k\left[\left[X_{1}, X_{2}, X_{3}, Y_{1}, Y_{2}, Y_{3}, Y_{4}\right]\right]$ and let $f \in\left(J: Y_{4}\right)$. Since

$$
\begin{aligned}
\left(J: Y_{4}\right)= & \left(X_{1} Y_{1}+X_{2} Y_{2}+X_{3} Y_{3}, Y_{1} Y_{2}-X_{3} Y_{4}, Y_{2} Y_{3}-X_{1} Y_{4}, Y_{1} Y_{3}-X_{2} Y_{4}\right): \\
& Y_{4}+\left(Y_{1}, \cdots, Y_{4}\right)
\end{aligned}
$$

we may assume that $f$ belongs to the first ideal on the right side. Let us write

$$
\begin{aligned}
f Y_{4}= & \left(g_{1}+g_{1}^{\prime} Y_{4}\right)\left(X_{1} Y_{1}+X_{2} Y_{2}+X_{3} Y_{3}\right)+\left(g_{2}+g_{2}^{\prime} Y_{4}\right)\left(Y_{1} Y_{2}-X_{3} Y_{4}\right) \\
& +\left(g_{3}+g_{3}^{\prime} Y_{4}\right)\left(Y_{2} Y_{3}-X_{1} Y_{4}\right)+\left(g_{4}+g_{4}^{\prime} Y_{4}\right)\left(Y_{1} Y_{3}-X_{2} Y_{4}\right),
\end{aligned}
$$

where $g_{i} \in k\left[\left[X_{1}, X_{2}, X_{3}, Y_{1}, Y_{2}, Y_{3}\right]\right]$. From this we see that

$$
g_{1}\left(X_{1} Y_{1}+X_{2} Y_{2}+X_{3} Y_{3}\right)+g_{2} Y_{1} Y_{2}+g_{3} Y_{2} Y_{3}+g_{4} Y_{1} Y_{3}=0
$$

and

$$
f \equiv-g_{2} X_{3}-g_{3} X_{1}-g_{4} X_{4} \bmod \left(Y_{1}, \cdots, Y_{4}\right) .
$$

From (I) we have

$$
Y_{1}\left(g_{1} X_{1}+g_{2} Y_{2}+g_{4} Y_{3}\right)+Y_{2}\left(g_{1} X_{2}+g_{3} Y_{3}\right)=0 .
$$

Since $Y_{1}, Y_{2}$ is a regular sequence in $R$ we have

$$
\begin{aligned}
& g_{1} X_{1}+g_{2} Y_{2}+g_{4} Y_{3}=h Y_{2} \\
& g_{1} X_{2}+g_{3} Y_{3}=-h Y_{1}
\end{aligned}
$$

for some $h \in R$. Since $X_{1}, Y_{2}, Y_{3}$ and $X_{2}, Y_{1}, Y_{3}$ are regular sequences in $R$ there are elements $a_{1}, a_{2}, a_{3}, b_{1}, b_{2}, b_{3}$ of $R$ such that

$$
\left(g_{1}, g_{2}-h, g_{4}\right)=\left(X_{1}, Y_{2}, Y_{3}\right)\left(\begin{array}{ccc}
0 & a_{1} & a_{2} \\
-a_{1} & 0 & a_{3} \\
-a_{2} & -a_{3} & 0
\end{array}\right)
$$




$$
\left(g_{1}, h, g_{3}\right)=\left(X_{2}, Y_{1}, Y_{3}\right)\left(\begin{array}{ccc}
0 & b_{1} & b_{2} \\
-b_{1} & 0 & b_{3} \\
-b_{2} & -b_{3} & 0
\end{array}\right)
$$

Hence

$$
g_{1}=-a_{1} Y_{2}-a_{2} Y_{3}=-b_{1} Y_{1}-b_{2} Y_{3} .
$$

Since $\operatorname{ch}(k)=2$ we have

$$
\left.\begin{array}{rl}
a_{2}+b_{2} & \equiv 0 \\
a_{1} & \equiv 0 \\
b_{1} & \equiv 0
\end{array}\right\} \bmod \left(Y_{1}, \cdots, Y_{4}\right) .
$$

By (II), (III) and (IV) we obtain

$$
\begin{aligned}
f & \equiv a_{1} X_{1} X_{3}+b_{1} X_{2} X_{3}+\left(a_{2}+b_{2}\right) X_{1} X_{2} \\
& \equiv 0 \quad \bmod \left(Y_{1}, \cdots, Y_{4}\right) .
\end{aligned}
$$

Proof of Example (2). By Lemma (4.3) we have $\left(0: y_{4}\right)=\left(y_{1}, \cdots, y_{4}\right)$. From the exact sequence

$$
0 \longrightarrow A /\left(0: y_{4}\right) \longrightarrow A \longrightarrow A / y_{4} A \longrightarrow 0
$$

we get $e(A)=e\left(A /\left(0: y_{4}\right)\right)+e\left(A / y_{4} A\right)$. Since $A / y_{4} A$ is isomorphic to the local ring in Example (1) and since $A /\left(0: y_{4}\right)$ is a regular local ring we have $e(A)=4$. It is easy to see that $x_{1}, x_{2}, x_{3}$ is a system of parameters of $A$ and that $\left(\left(x_{1}, x_{2}\right): x_{3}\right)=\left(x_{1}, x_{2}, y_{3}\right),\left(\left(x_{2}, x_{3}\right): x_{1}\right)=\left(x_{2}, x_{3}, y_{1}\right)$ and $\left(\left(x_{1}, x_{3}\right): x_{2}\right)$ $=\left(x_{1}, x_{3}, y_{2}\right)$. This shows that $A$ is not Cohen-Macaulay. It is easy to verify that $m^{2}=\left(x_{1}, x_{2}, x_{3}\right) m$. By Lemma (4.1) we see that $R(m)$ is CohenMacaulay. By Theorem (3.1) it is enough to show that $\mathscr{K}_{G(m)}=G(m)(-2)$. Since $A$ is defined by homogeneous polynomials

$$
G(m)=k\left[X_{1}, X_{2}, X_{3}, Y_{1}, Y_{2}, Y_{3}, Y_{4}\right] / J^{*}=k\left[x_{1}, x_{2}, \cdots, y_{4}\right],
$$

where $J^{*}$ is generated by the polynomials generating $J$. Let $S=k\left[x_{1}, x_{2}, x_{3}\right]$. Then $S$ is a polynomial ring with $\operatorname{dim} S=3$ and $G(m)$ is generated by 1 , $y_{1}, y_{2}, y_{3}, y_{4}$ as an $S$-module. Since $G(m)$ has rank 4 as an $S$-module and depth $G(m)=2$ we get a finite free resolution of $G(m)$ as $S$-module

$$
0 \longrightarrow S(-2) \underset{\left[0, x_{1}, x_{2}, x_{3}, 0\right]}{\longrightarrow} S \oplus S^{4}(-1) \underset{d}{\longrightarrow} G(m) \longrightarrow 0,
$$

where $d$ is given by $d\left(e_{0}\right)=1$ and $d\left(e_{i}\right)=y_{i}$ for $1 \leq i \leq 4$, with suitable free basis $e_{0}, e_{1}, \cdots, e_{4}$ of $S \oplus S^{4}(-1)$ with $\operatorname{deg}\left(e_{0}\right)=0$ and $\operatorname{deg}\left(e_{i}\right)=1$ for 
$1 \leq i \leq 4 . \quad$ By Corollary (1.11) $\mathscr{K}_{G(m)}=\mathscr{H}_{o m_{S}}(G(m), S(-3))$

The $G(m)$-structure of $\mathscr{K}_{G(m)}$ is given by

$$
(x f)(y)=f(x y) \quad \text { for } f \in \mathscr{H}_{\mathrm{om}_{S}}(G(m), S(-3)) \text { and } x, y \in G(m) .
$$

$\mathscr{K}_{G(m)}$ is generated by $e_{0}^{*}, x_{2} e_{3}^{*}-x_{3} e_{2}^{*}, x_{3} e_{1}^{*}-x_{1} e_{3}^{*}, x_{1} e_{2}^{*}-x_{2} e_{1}^{*}$ and $e_{4}^{*}$ as an $S$-module, where $e_{i}^{*}$ is the dual base of $e_{i}$ with $\operatorname{deg}\left(e_{0}^{*}\right)=3$ and $\operatorname{deg}\left(e_{i}^{*}\right)=2$ for $1 \leq i \leq 4$. Using the fact that $\operatorname{ch}(k)=2$ we can easily verify the following relations as $G(m)$-module.

$$
\begin{aligned}
& y_{4} e_{4}^{*}=e_{0}^{*} \\
& y_{1} e_{4}^{*}=x_{2} e_{3}^{*}-x_{3} e_{2}^{*} \\
& y_{2} e_{4}^{*}=x_{3} e_{1}^{*}-x_{1} e_{3}^{*} \\
& y_{3} e_{4}^{*}=x_{1} e_{2}^{*}-x_{2} e_{1}^{*}
\end{aligned}
$$

Hence $\mathscr{K}_{G(m)}=G(m)(-2)$ and hence $R(m)$ is Gorenstein by Theorem (3.1).

Example (3). Let $A$ be same as in Example (2). We put $B=$ $A\left[\left[T_{1}, \cdots, T_{n}\right]\right]$, where $T_{1}, \cdots, T_{n}$ are indeterminates over $A$. Let $I=m B$. Then $R(I)=R(m) \otimes_{A} B$ is Gorenstein since $B$ is faithfully flat over $A$. If $n \geq 3$ we have $2 \mathrm{ht}(I)=6 \leq \operatorname{dim} B$. But $B$ is not Gorenstein.

Remark. a) If in Example (2) $\operatorname{ch}(k) \neq 2 A$ is not Buchsbaum. This can be seen as follows. If $A$ is Buchsbaum we have

$$
\begin{aligned}
e(A) & =l_{A}\left(A /\left(x_{1}, x_{2}, x_{3}\right)\right)-l_{A}\left(\left(x_{1}, x_{2}\right): x_{3} /\left(x_{1}, x_{2}\right)\right) \\
& =5-1=4 .
\end{aligned}
$$

On the other hand one can easily see $\left(0: y_{4}\right) \supset\left(y_{1}, \cdots, y_{4}, x_{1} x_{2}\right)$ and $\operatorname{dim} A /\left(0: y_{4}\right)<3$. This implies $e(A)=e\left(A / y_{4} A\right)=3$, a contradiction.

b) Example (3) shows that Corollary (3.8) is false without any restriction on the local cohomology modules of $A$.

\section{REFERENCES}

[A] Y. Aoyama, Some basic results on canonical modules, J. Math. Kyoto Univ., 23 (1983), 85-94.

[AG] Y. Aoyama and S. Goto, On the type of graded Cohen-Macaulay rings, J. Math. Kyoto Univ., 15 (1975), 275-284.

[B] H. Bass, On the ubiquity of Gorenstein rings, Math. Z., 82 (1963), 8-28.

[ $\left.\mathrm{Gr}_{1}\right]$ A. Grothendieck, Sur quelque points d'algebre homologique, Tohoku Math. J., IX (1957), 119-221.

$\left[\mathrm{Gr}_{2}\right]-$ Local cohomology, Lect. Notes in Math., 41, Berlin-Heidelberg-New York, 1967. 
[GS] S. Goto and Y. Shimoda, On the Rees algebras of Cohen-Macaulay local rings, Commutative algebra (analytic methods), Lecture Notes in Pure and Applied Mathematics, 68 (1982), 201-231.

[GW] S. Goto and K. Watanabe, On graded rings I, J. Math. Soc. Japan, 30 (1978), 179-213.

[HI] M. Herrmann and S. Ikeda, Remarks on lifting of Cohen-Macaulay property, Nagoya Math. J., 92 (1983), 121-132.

[HK] J. Herzog and E. Kunz, Der kanonische Modul eines Cohen-Macaulay Rings, Springer Lect. Notes in Math., 238 (1971).

[ $\left.\mathrm{I}_{1}\right] \quad \mathrm{S}$. Ikeda, The Cohen-Macaulayness of the Rees algebras of local rings, Nagoya Math. J., 89 (1983), 47-63.

$\left[\mathrm{I}_{2}\right]$ - Remarks on Rees algebras and graded rings with multiplicity 3, Preprint.

[M] E. Matlis, Injective modules over Noetherian rings, Pacific J. Math., 8 (1958), $511-528$.

[MR] J. Matijevic and P. Roberts, A conjecture of Nagata on graded Cohen-Macaulay rings, J. Math. Kyoto Univ., 14 (1974) 125-128.

[NR] D. G. Northcott and D. Rees, Reductions of ideals in local rings, Proc. Camb. Phil. Soc., 50 (1954), 145-158.

[CST] N. T. Cuong, P. Schenzel and N. V. Trung, Verallgemeinerte Cohen-MacaulayModuln, Math. Nachr., 85 (1978), 57-73. 